Pacific Journal of Mathematic 


\title{
A GENERALIZATION OF COMMUTATIVE AND ASSOCIATIVE RINGS
}

\author{
ERWIN KLEINFELD
}

Let $R$ be a ring satisfying the following three defining relations: (i) $\left(x, y^{2}, x\right)=y \circ(x, y, x)$, (ii) $(x, y, z)+(y, z, x)+(z$, $x, y)=0$, and (iii) $((x, y), x, x)=0$, where $(a, b, c)=(a b) c-a(b c)$, $(a, b)=a b-b a$, and $a \circ b=a b+b a$. All three identities follow from commutativity, hence are true in Jordan rings. Besides (i) holds in Lie and alternative rings, (ii) holds in Lie and quasiassociative rings and in alternative rings of characteristic three, while (iii) holds in right alternative rings. The main result is that if $R$ has characteristic $\neq 2,3$ (that means no elements in $R$ have additive order two or three) and no divisors of zero then $R$ must be either associative or commutative.

The classification of commutative rings has not been attempted, perhaps because the important tool of decomposition relative to an idempotent due to Albert requires power-associativity. It is well known that commutative rings need not be fourth power associative. Besides one would have to find a way to classify the numerous finite, commutative devision rings. The choice of identities was dictated by the fact that there exist rings without divisors of zero which satisfy (i) and (ii) but which are not commutative. The Cayley-Dickson division algebras satisfy (i) and (iii) yet are not commutative. In fact the Cayley-Dickson division algebras over fields of characteristic 3 satisfy all three identities. There is some reason to believe that ultimately there will be found a set of identities suitable for generalizing the better known rings such as alternative, Jordan and Lie rings. The present study is helpfull in delineating possible candidates for replacing commutativity. A few examples will be discussed at the end of the paper.

Throughout most of the paper we shall require $R$ to be a ring which satisfies (i)-(iii), has characteristic $\neq 2,3$ and no divisors of zero. However in the beginning we can dispense with (iii) and weaken the assumption of no divisors of zero to assuming that there exists no element $x \neq 0$, such that $x^{2}=0$. In every ring we have the Teichmüller identity

$$
\begin{aligned}
f(w, x, y, z)=(w x, y, z) & -(w, x y, z)+(w, x, y z) \\
& -w(x, y, z)-(w, x, y) z=0
\end{aligned}
$$




$$
\begin{aligned}
0= & f(w, x, y, z)-f(x, y, z, w)+f(y, z, w, x)-f(z, w, x, y) \\
= & (w x, y, z)-(w, x y, z)+(w, x, y z)-w(x, y, z)-(w, x, y) z \\
& -(x y, z, w)+(x, y z, w)-(x, y, z w)+x(y, z, w)+(x, y, z) w \\
& +(y z, w, x)-(y, z w, x)+(y, z, w x)-y(z, w, x)-(y, z, w) x \\
& -(z w, x, y)+(z, w x, y)-(z, w, x y)+z(w, x, y)+(z, w, x) y
\end{aligned}
$$

As a result of (ii) we have $(w x, y, z)+(z, w x, y)+(y, z, w x)=0$,

$$
-(x y, z, w)-(w, x y, z)-(z, w, x y)=0,
$$

$(y z, w, x)+(x, y z, w)+(w, x, y z)=0$, and $-(z w, x, y)-(y, z w, x)-$ $(x, y, z w)=0$. Comparing these identities with the previous one we see that $-w(x, y, z)-(w, x, y) z+x(y, z, w)+(x, y, z) w-y(z, w, x)-$ $(y, z, w) x+z(w, x, y)+(z, w, x) y=0$. Hence

$$
(w,(x, y, z))-(x,(y, z, w))+(y,(z, w, x))-(z,(w, x, y))=0 .
$$

In (2) put $w=z=x$. Then $0=(x,(x, y, x))-(x,(x, x, y))+(y,(x, x, x))-$ $(x,(y, x, x))$. If $y=z=x$ in (ii) then $3(x, x, x)=0$, so that $(x, x, x)=0$. If $z=x$ in (ii) then $(x, y, x)+(x, x, y)+(y, x, x)=0$. This results in $2(x,(x, y, x))=0$, so that

$$
(x,(x, y, x))=0 \text {. }
$$

In an arbitrary ring we have the semi-Jacobi identity $(x y, z)-x(y, z)-$ $(x, z) y=(x, y, z)+(z, x, y)-(x, z, y)$. But in view of (ii) we may rewrite it as

$$
(x y, z)-x(y, z)-(x, z) y=-(y, z, x)-(x, z, y) .
$$

Define $u=(x, y, x)$. With this notation (3) states that $(u, x)=0$. Then as a result of (4) $(u x, x)=-(u, x, x)-(x, x, u)$. But (ii) implies that $-(u, x, x)-(x, x, u)=(x, u, x)$, so that $(u x, x)=(x, u, x)$. Similarly $(x u, x)=-(x, x, u)-(u, x, x)=(x, u, x)$, so that $(x \circ u, x)=2(x, u, x)$. From a linearization of (i) it follows that $(x, x \circ y, x)=x \circ(x, y, x)+$ $y \circ(x, x, x)=x \circ(x, y, x)$. Then because of $(3), \quad 0=(x,(x, x \circ y, x))=$ $(x, x \circ u)$. Consequently $2(x, u, x)=0$, so that

$$
(x, u, x)=(x,(x, y, x), x)=0 .
$$

Substituting $y^{2}$ for $y$ in (5) we see that $0=\left(x,\left(x, y^{2}, x\right), x\right)=(x, y \circ u, x)$, using (i). From a linearization of (i) it follows that $(x, y \circ u, x)=$ $y \circ(x, u, x)+u \circ(x, y, x)=u \circ(x, y, x)$, using (5). Thus $0=u \circ(x, y, x)$. But $(x, y, x)=u$, so that $2 u^{2}=0$. But then $u^{2}=0$, so that $u=$ $(x, y, x)=0$. We have proved

Lemma. If $R$ is a ring of characteristic $\neq 2,3$, satisfies (i) and 
(ii) and has no element $x \neq 0$, such that $x^{2}=0$, then $R$ must be flexible, that is satisfy the identity $(x, y, x)=0$.

For the remainder of the paper we assume that $R$ satisifes (iii), has no divisors of zero and satisfies the hypotheses of the Lemma. In view of the Lemma, (4) may be rewritten as

$$
(x y, z)=x(y, z)+(x, z) y \text {. }
$$

Using (6) repeatedly we have $((w, x, y), z)=(w x \cdot y-w \cdot x y, z)=$ $w x \cdot(y, z)+w(x, z) \cdot y+(w, z) x \cdot y-w \cdot x(y, z)-w \cdot(x, z) y-(w, z) \cdot x y=$ $((w, z), x, y)+(w,(x, z), y)+(w, x,(y, z))$. Thus

$$
((w, x, y), z)=((w, z), x, y)+(w,(x, z), y)+(w, x,(y, z)) .
$$

If we put $z=y=x$ in $(7)$ then $((w, x, x), x)=((w, x), x, x)+(w,(x, x), x)+$ $(w, x,(x, x))$. As a result of (iii) we have

$$
((w, x, x), x)=0=((x, x, w), x) .
$$

Let $v=(x, y)$. Then $0=f(x, y, x, y)=(x y, x, y)-(x, y x, y)+(x, y, x y)-$ $x(y, x, y)-(x, y, x) y$. Because of the Lemma the last two terms vanish. Then (ii) implies that $(x y, x, y)+(x, y, x y)=-(y, x y, x)=$ $(x, x y, y)$, using the Lemma. Hence $0=(x, x y, y)-(x, y x, y)=(x, v, y)$. We have shown that

$$
(x, v, y)=0 \text {. }
$$

Linearizing (iii) we see that $((y, y), x, x)+((x, y), y, x)+((x, y), x, y)=0$. Thus $(v, y, x)+(v, x, y)=0$. Since (ii) implies $(v, y, x)+(y, x, v)+$ $(x, v, y)=0$, we may use (9) to obtain $(v, y, x)+(y, x, v)=0$. But then $(v, x, y)=(y, x, v)$. Because of the Lemma we have $(v, x, y)=$ $-(y, x, v)$. Thus $(v, x, y)=0=(y, x, v)$. This together with the Lemma and (9) implies, using (ii)

$$
\begin{aligned}
(x, v, y) & =(y, v, x)=(v, x, y)=(y, x, v)=(v, y, x) \\
& =(x, y, v)=0 .
\end{aligned}
$$

In (7) put $w=x$, and $z=y$. Then $((x, x, y), y)=((x, y), x, y)+$ $(x,(x, y), y)+(x, x,(y, y))=0$, using $(10)$. Hence

$$
((x, x, y), y)=0=((y, x, x), y) .
$$

By using a linearization of (11) together with flexibility it follows that

$$
(x,(x, y, z))=-(x,(x, z, y))=(x,(y, z, x))=-(x,(z, y, x)) .
$$

But since $(x, y, z)+(y, z, x)+(z, x, y)=0$, using (ii) we have 


$$
(x,(z, x, y))=-2(x,(x, y, z)) .
$$

Using (6) we see that $\left(x^{2},(x, x, y)\right)=x \circ(x,(x, x, y))=0$, as a consequence of (8). But then a linearization of (11) gives us $\left(\left(x, x, x^{2}\right), y\right)=$ $-\left((x, x, y), x^{2}\right)=\left(x^{2},(x, x, y)\right)=0$. So

$$
\left(\left(x^{2}, x, x\right), y\right)=0 \text {. }
$$

By linearizing (14) we see that

$$
((x \circ y, x, x), y)+\left(\left(x^{2}, y, x\right), y\right)+\left(\left(x^{2}, x, y\right), y\right)=0 .
$$

However a linearization of (11) shows that

$$
((x \circ y, x, x), y)=-((y, x, x), x \circ y)=(x \circ y,(y, x, x))=-(x \circ y,(x, x, y)) .
$$

But use of (6), together with (8) and (11) implies that $(x \circ y,(x, x, y))=$ 0 , so that $((x \circ y, x, x), y)=0$. Hence $\left(\left(x^{2}, y, x\right), y\right)+\left(\left(x^{2}, x, y\right), y\right)=0$. But this last identy together with a linearization of (11) and the Lemma shows that $\left(\left(x^{2}, x, y\right), y\right)=-\left(\left(x^{2}, y, x\right), y\right)=\left(\left(x, y, x^{2}\right), y\right)=-\left(\left(y, x, x^{2}\right), y\right)=$ $\left(\left(y, x^{2}, x\right), y\right)$. Since (ii) gives us $\left(x^{2}, x, y\right)+\left(x, y, x^{2}\right)+\left(y, x^{2}, x\right)=0$, we see that $3\left(\left(x^{2}, x, y\right), y\right)=0$. Using characteristic different from 3 , this leads to $\left(\left(x^{2}, x, y\right), y\right)=0$. Thus

$$
\begin{aligned}
\left(\left(x^{2}, x, y\right), y\right) & =\left(\left(x, y, x^{2}\right), y\right)=\left(\left(y, x^{2}, x\right), y\right)=\left(\left(x^{2}, y, x\right), y\right) \\
& =\left(\left(y, x, x^{2}\right), y\right)=\left(\left(x, x^{2}, y\right), y\right)=0
\end{aligned}
$$

Then $0=f(x, x, x, y)=\left(x^{2}, x, y\right)-\left(x, x^{2}, y\right)+(x, x, x y)-x(x, x, y)-$ $(x, x, x) y$ implies, using (15) that $((x, x, x y), y)=(x(x, x, y), y)$. Following (14) we proved that $((x, x, x y), y)=0$. Thus $(x(x, x, y), y)=0$. But then using (6) we have $0=(x(x, x, y), y)=x((x, x, y), y)+(x, y)$ $(x, x, y)=(x, y)(x, x, y)$, using (11). Thus

$$
(x, y)(x, x, y)=0 \text {. }
$$

Linearizing (10) we obtain

$$
((x, y), w, t)+((w, y), x, t)+((x, t), w, y)+((w, t), x, y)=0 .
$$

In $(17)$ put $w=(x, z)$, and $t=(x, x, z)$. Then $(x, t)=(x,(x, x, z))=0$, because of (8). Also using a linearization of (11) together with (iii), $(w, t)=((x, z),(x, x, z))=-(z,(x, \hat{x},(x, z))=0$. Thus the last two terms vanish and we are left with

$$
((x, y),(x, z),(x, x, z))=-\{((x, z), y), x,(x, x, z)) .
$$

We note that by using (6) twice we get

$$
((x, y), z)+((y, z), x)+((z, x), y)=0 .
$$


If we linearize $(10)$ we see that $((x, y), x, t)=-((x, t), x, y)$. In this last identity put $y=(y, z)$, and $t=(x, x, z)$. Then $((x,(y, z)), x,(x, x, z))=$ $-((x,(x, x, z)), x,(y, z))=0$, using $(8)$. Thus $(((y, z), x), x,(x, x, z))=0$. But then using $(19),-(((x, z), y), x,(x, x, z))=(((z, x), y), x,(x, x, z))=$ $-(((x, y), z), x,(x, x, z))$. This together with (18) implies that

$$
((x, y),(x, z),(x, x, z))=-(((x, y), z), x,(x, x, z)) .
$$

Linearizing (16) we see that

$$
\begin{gathered}
(x, y)(t, t, s)+(t, y)(x, t, s)+(t, y)(t, x, s)+(x, s)(t, t, y) \\
+(t, s)(x, t, y)+(t, s)(t, x, y)=0 .
\end{gathered}
$$

In (21) let $t=(x, y)$, and $s=(x, x, z)$. As a result of linearizing (11) we see that $(t, s)=((x, y),(x, x, z))=-(z,(x, x,(x, y)))=0$, using (iii). This makes the last two terms vanish. Also $(x, s)=(x,(x, x, z))=0$, because of (8), so only the first three terms remain. But $(x, t, s)=$ $(x,(x, y),(x, x, z))=-(x,(x,(x, x, z)), y)=0$, using a linearization of (10) together with (8). Similarly

$$
(t, x, s)=((x, y), x,(x, x, z))=-((x,(x, x, z)), x,(x, y))=0,
$$

again utilizing a linearization of (10) together with (8). Thus only one term survives, namely $0=(x, y)(t, t, s)=(x, y)((x, y),(x, y),(x, x, z))$. Using the hypothesis of no divisors of zero we get

$$
((x, y),(x, y),(x, x, z))=0 \text {. }
$$

Linearizing the last identity we get

$$
((x, y),(x, z),(x, x, z)=-((x, z),(x, y),(x, x, z)) .
$$

Using (17) on the right hand side of (22) we note that $-((x, z),(x, y)$, $(x, x, z))=(((x, y), z), x,(x, x, z))+((x,(x, x, z)),(x, y), z)+(((x, y),(x, x, z)), x, z)$. However the second term of the last identity vanishes because of (8), while $((x, y),(x, x, z))=-(z,(x, x,(x, y))=0$, because of a linearization of (11) and (iii). Thus the third term vanishes also. This leaves us with

$$
-((x, z),(x, y),(x, x, z))=(((x, y), z), x,(x, x, z)) .
$$

Combining (22) with (23) we find that

$$
((x, y),(x, z),(x, x, z))=(((x, y), z), x,(x, x, z)) .
$$

But comparing (24) and (20) and use of characteristic not 2 yields

$$
((x, z),(x, y),(x, x, z))=0=((x, y),(x, z),(x, x, z)) \text {. }
$$


Using (25), flexibility and (ii), it follows that

$$
\begin{aligned}
& ((x, x, z),(x, z),(x, y))=((x, x, z),(x, y),(x, z))=0 \\
= & ((x, y),(x, x, z),(x, z))=((x, z),(x, x, z),(x, y)) .
\end{aligned}
$$

In the course of proving (16) we established $(x(x, x, y), y)=0$. Then use of (8) and (6) shows that $0=((x, x, y) x, y)=(x, x, y)(x, y)$, because of (11). Using this latest identity together with (26) gives us $0=$ $((x, y),(x, x, z),(x, z))=[(x, y)((x, x, z)](x, z)$. But a linearization of $(16)$ shows that $(x, y)(x, x, z)=-(x, z)(x, x, y)$, so that $0=-[(x, z)(x, x, y)](x, z)$. Again using the hypothesis of no divisors of zero, it is clear that

$$
(x, z)(x, x, y)=0 \text {. }
$$

Consequently either $(x, z)=0$, for every $z$ in $R$ or $(x, x, y)=0$, for all $y$ in $R$. In particular if we assume that $(x, y) \neq 0$, then both $(x, x, z)=0$, and $(y, y, z)=0$, for all $z$ in $R$. Since $(x, x+y)=(x, y) \neq$ 0 , then $0=(x+y, x+y, z)=(x, y, z)+(y, x, z)$. Using flexibility and (ii) it follows that

$$
\begin{gathered}
(x, y, z)=-(y, x, z)=(z, x, y)=-(z, y, x), \\
(x, z, y)=2(x, y, z)=-(y, z, x) .
\end{gathered}
$$

From a linearization of (16) we have $(x, y)(x, y, z)+(x, y)(x, z, y)+$ $(x, z)(x, y, y)=0$. Since $(x, y) \neq 0$, and $(x, y, y)=0$, we get $(x, y, z)=$ $-(x, z, y)$. This together with (29) leads to $3(x, y, z)=0$, so that $(x, y, z)=0$. Similarly $(y, z, x)=0$, and $(z, x, y)=0$. Thus if $(x, y) \neq$ 0 , then $(x, y, z)=(z, x, y)=(y, z, x)=0$. Hence

$$
0=(x, y)(x, y, z)=(x, y)(y, z, x)=(x, y)(z, x, y) \text {. }
$$

Linearize $(30)$ to obtain $(x, y)(x, r, s)=-(x, r)(x, y, s)$. Assume $(x, y) \neq$ 0 . Then (30) implies that $(x, y, s)=0$. But then $(x, y)(x, r, s)=0$. Similarly $(x, y)(r, s, x)=0$, and $(x, y)(s, x, r)=0$. Using the hypothesis of no divisors of zero then $x$ lies in the nucleus $N$ of $R$.

$$
\text { If }(x, y) \neq 0 \text {, then } x, y \text { belong to the nucleus } N \text { of } R \text {. }
$$

Suppose $n, n^{\prime}$ belong to $N$ while $a, b, c$ are arbitrary elements of $R$. Then because of a linearization of $(30)$ we have $\left(n, n^{\prime}\right)(a, b, c)+$ $\left(a, n^{\prime}\right)(n, b, c)+(n, b)\left(a, n^{\prime}, c\right)+(a, b)\left(n, n^{\prime}, c\right)=0=\left(n, n^{\prime}\right)(a, b, c)$. If $R$ is not associative then $\left(n, n^{\prime}\right)=0$. Thus suppose there exist $x, y$ in $R$ such that $(x, y) \neq 0$. As a result of (31) $x, y$ belong to $N$. If $R$ is not associative then $(x, y)=0$, contrary to assumption. Hence $R$ must be associative. We have proved 
THEOREM. If $R$ is a ring of characteristic $\neq 2,3$ that satisfies (i)-(iii) and has no divisors of zero then $R$ is either commutative or associative.

We conclude with a short discussion of the identities and some examples. If we take the ring $Q$ of real quaternions and define a new product $x * y=c(x y)+(1-c)(y x)$, where $c$ is a scalar, $c \neq 1 / 2$, then $Q(*)$ becomes a ring that is flexible, satisfies (ii) and is even a noncommutative Jordan ring. It may also be verified that $Q(*)$ has no divisors of zero. But it does not satisfy (iii) and in fact is neither associative nor commutative. The Cayley-Dickson division algebras satisfy (i) and (iii), and those of characteristic 3 satisfy even (ii), yet are neither associative nor commutative. Hence we could not omit either (ii) or (iii) from the hypothesis.

Received September 2, 1970. This work was supported in part by the National Science Foundation (NSF Grant GP 23403).

THE UNIVERSITY OF IOWA 



\section{PACIFIC JOURNAL OF MATHEMATICS}

\section{EDITORS}

H. SAMELSON

Stanford University

Stanford, California 94305

C. R. Hовву

University of Washington

Seattle, Washington 98105
J. DUGUNDJI

Department of Mathematics

University of Southern California

Los Angeles, California 90007

RICHARD ARENS

University of California

Los Angeles, California 90024

\section{ASSOCIATE EDITORS}
E. F. BECKENBACH
B. H. NeumanN
F. WOLF
K. YoSHIDA

\section{SUPPORTING INSTITUTIONS}

UNIVERSITY OF BRITISH COLUMBIA

CALIFORNIA INSTITUTE OF TECHNOLOGY

UNIVERSITY OF CALIFORNIA

MONTANA STATE UNIVERSITY

UNIVERSITY OF NEVADA

NEW MEXICO STATE UNIVERSITY

OREGON STATE UNIVERSITY

UNIVERSITY OF OREGON

OSAKA UNIVERSITY

UNIVERSITY OF SOUTHERN CALIFORNIA
STANFORD UNIVERSITY

UNIVERSITY OF TOKYO

UNIVERSITY OF UTAH

WASHINGTON STATE UNIVERSITY

UNIVERSITY OF WASHINGTON

AMERICAN MATHEMATICAL SOCIETY CHEVRON RESEARCH CORPORATION NAVAL WEAPONS CENTER 


\section{Pacific Journal of Mathematics}

\section{Vol. 38, No. $1 \quad$ March, 1971}

Bruce Alan Barnes, Banach algebras which are ideals in a Banach algebra ..... 1

David W. Boyd, Inequalities for positive integral operators............... 9

Lawrence Gerald Brown, Note on the open mapping theorem .............. 25

Stephen Daniel Comer, Representations by algebras of sections over Boolean

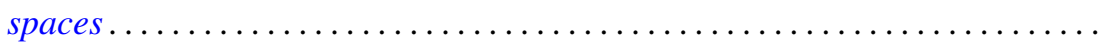

John R. Edwards and Stanley G. Wayment, On the nonequivalence of

conservative Hausdorff methods and Hausdorff moment sequences ........

P. D. T. A. Elliott, On the limiting distribution of additive functions $(\bmod 1) \ldots \ldots$

Mary Rodriguez Embry, Classifying special operators by means of subsets

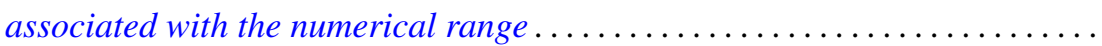

Darald Joe Hartfiel, Counterexamples to a conjecture of G. N. de Oliveira ......

C. Ward Henson, A family of countable homogeneous graphs...............

Satoru Igari and Shigehiko Kuratsubo, A sufficient condition for

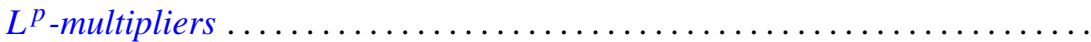

William A. Kirk, Fixed point theorems for nonlinear nonexpansive and

generalized contraction mappings............................

Erwin Kleinfeld, A generalization of commutative and associative rings ...... 95

D. B. Lahiri, Some restricted partition functions. Congruences modulo $11 \ldots \ldots 103$

T. Y. Lin, Homological algebra of stable homotopy ring $\pi *$ of spheres ....... 117

Morris Marden, A representation for the logarithmic derivative of a meromorphic function...........................

John Charles Nichols and James C. Smith, Examples concerning sum properties for metric-dependent dimension functions . .

Asit Baran Raha, On completely Hausdorff-completion of a completely

Hausdorff space.

M. Rajagopalan and Bertram Manuel Schreiber, Ergodic automorphisms and affine transformations of locally compact groups..........

N. V. Rao and Ashoke Kumar Roy, Linear isometries of some function

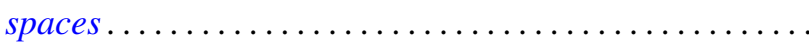

William Francis Reynolds, Blocks and F-class algebras of finite groups

Richard Rochberg, Which linear maps of the disk algebra are multiplicative ...

Gary Sampson, Sharp estimates of convolution transforms in terms of decreasing

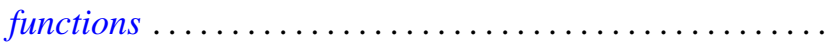

Stephen Scheinberg, Fatou's lemma in normed linear spaces

Ken Shaw, Whittaker constants for entire functions of several complex

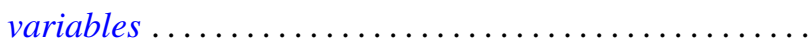

James DeWitt Stein, Two uniform boundedness theorems................ 251

$\mathrm{Li} \mathrm{Pi} \mathrm{Su,} \mathrm{Homomorphisms} \mathrm{of} \mathrm{near-rings} \mathrm{of} \mathrm{continuous} \mathrm{functions} \mathrm{.} \mathrm{.............} 261$

Stephen Willard, Functionally compact spaces, $C$-compact spaces and mappings of minimal Hausdorff spaces....................... 